\title{
Nasal Schwannoma
}

\author{
https://doi.org/10.47210/bjohns.2020.v28i3.311
}

Surinder K Singhal, ${ }^{1}$ Ankit Gulati, ${ }^{1}$ Nitin Gupta, ${ }^{1}$ Mugdha Singh ${ }^{1}$

\section{Introduction}

\section{ABSTRACT}

Congenital deafness in a child is often missed. Several distraction tests have evolved over time to diagnose congenital deafness. A schwannoma is a benign nerve sheath tumuor of myelinated nerves arising from Schwann cells. In the head and neck region, the most common site is the eighth cranial nerve (vestibulocochlear). Only 4\% of schwannomas seen in the head and neck region arise from the nose and paranasal sinuses involving branches of the trigeminal nerve (ophthalmic or maxillary) or from the autonomic nervous system.

Case Report

A 29 year old female patient presented to the Ear, Nose and Throat Out Patient Department with the complaints of left sided nasal obstruction and left sided nasal bleed. On anterior rhinoscopy, a single, smooth, greyish, non-pulsatile polypoidal mass was seen in the left nasal cavity seeming to be arising medial to middle turbinate. A provisional diagnosis of benign nasal mass was made and the patient underwent excision under general anaesthesia. On histopathology, an impression of Schwannoma was made.

\section{Discussion}

Sino-nasal schwannomas are a very rare entity with non specific imaging studies. A confirmatory diagnosis can be made only after histopathology. The treatment modality of choice is surgical excision of the mass, taking care to leave no residual, so as to prevent a recurrence.

$\underline{\text { Kevwords }}$

Schwannoma; Neurilemoma; Nose

A schwannoma is a benign nerve sheath tumour of myelinated nerves arising from Schwann cells. In the head and neck region, the most common site is the eighth cranial nerve (vestibulocochlear); other sites include the scalp, face, parotid gland, oral cavity, pharynx, larynx, and trachea. Only $4 \%$ of schwannomas seen in the head and neck region arise from the nose and paranasal sinuses involving branches of the trigeminal nerve (ophthalmic or maxillary) or from the autonomic nerv-ous system. ${ }^{1}$ Confirmatory diagnosis can only be made with the help of histopathology.

We are reporting a rare case of Nasal Schwannoma encountered and dealt with at our institution.

\section{Case Report}

A29 year old female presented to the Ear, Nose and Throat (ENT) Department with the complaint of left sided nasal obstruction for the last 18 months which was insidious in onset, progressive, persistent, not relieved with medications and associated with left sided ear fullness. No diurnal variation was associated with the complaint. It was associated with left sided nasal discharge which was mucopurulent, yellowish in colour, thick in consistency and non foul smelling. There was a history of left sided nasal bleed twice in the past six months, both episodes were sudden in onset, approximately $30 \mathrm{ml}$ in amount and relieved spontaneously. There was no history of excessive sneezing, facial pain or pressure or any disturbance in olfaction or vision. There was no history of fever or trauma and there were no other otorhinolaryngological complaints. The past, personal and family histories were insignificant. External examination of the nose revealed no abnormalities.

1 - Department of ENT, Government Medical College and Hospital, Sector 32, Chandigarh

Corresponding author:

Dr Ankit Gulati

email: gulati.ankit07@gmail.com 


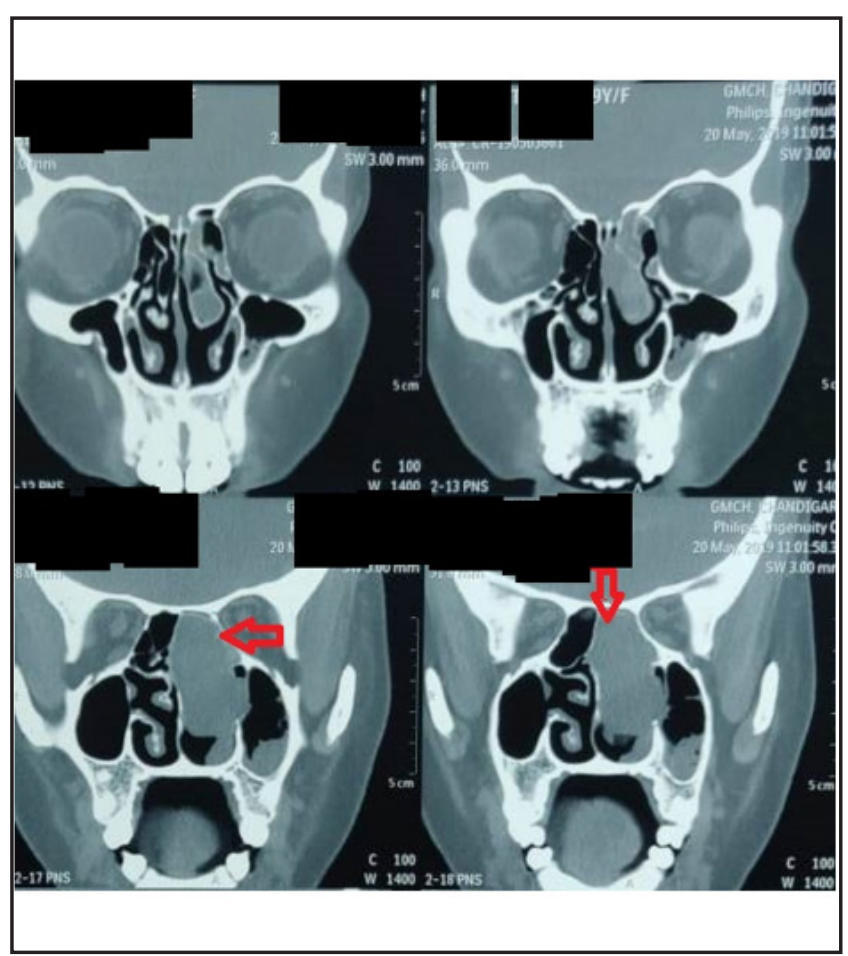

Fig. 1. coronal cut showing soft tissue density in the left nasal cavity, ethmoids and sphenoid (red arrow)

On anterior rhinoscopy; mucoid discharge was seen on the floor on left side and was suctioned. A single, smooth, greyish, non pulsatile polypoidal mass was seen in the left nasal cavity seem to be arising medial to middle turbinate. On probing, it was firm, non tender, bled slightly on touch. Posterior rhinoscopy revealed a single greyish mass in the left side of nasopharynx. Cold spatula test showed decreased misting on the left side and cotton wool test showed decreased movement of the wisp on the left. Paranasal sinus examination was within normal limits. Bilateral tympanic membranes were intact and normal.

The rest of the otorhinolaryngological and head and neck examination was unremarkable. The patient underwent non contrast computed tomography scan of the nose and paranasal sinuses which showed homogenous soft tissue density filling the left nasal cavity reaching upto the posterior choana, left anterior and posterior ethmoids, bilateral sphenoid sinuses. Minimal soft tissue density was also seen in the left maxillary sinus (Fig. 1). The patient then underwent

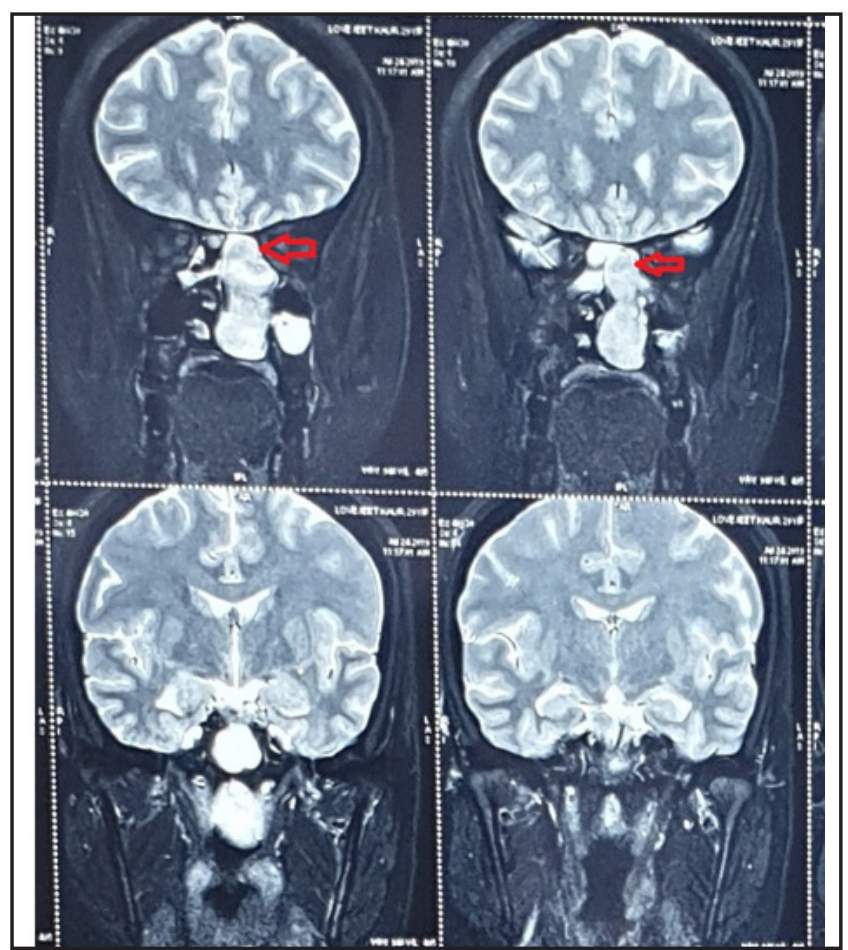

Fig. 2. MRI depicting the lesion in the left nasal cavity (red arrow)

contrast enhanced magnetic resonance imaging of the paranasal sinuses which revealed a large, approximately 57.4(AP) x 21.7(TR) x 46.9(CC) $\mathrm{mm}$, heterogenous $\mathrm{T} 2$ hyper intense soft tissue lesion in the left ethmoidal air cells, left nasal cavity and nasopharynx. It was seen to block the sphenoethmoidal recess superiorly and the left maxillary ostium laterally (Fig. 2).

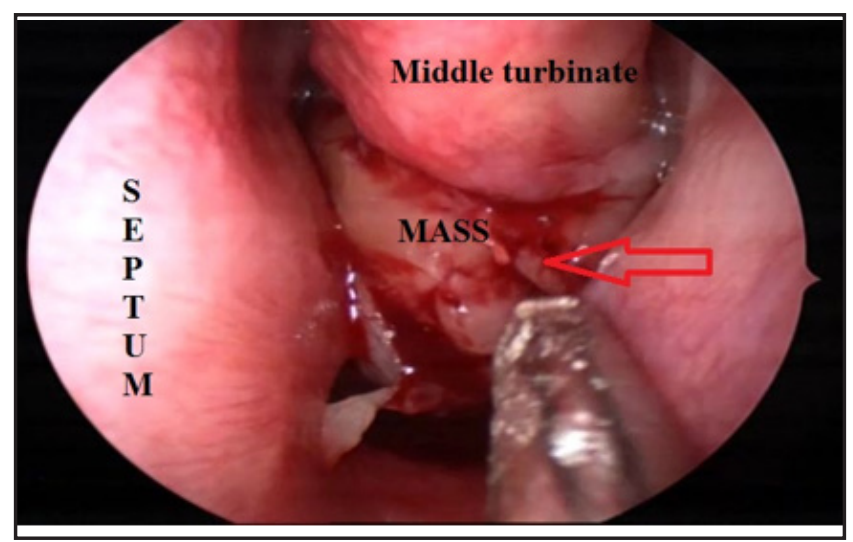

Fig. 3. Intra-operative picture of the mass (red arrow) 


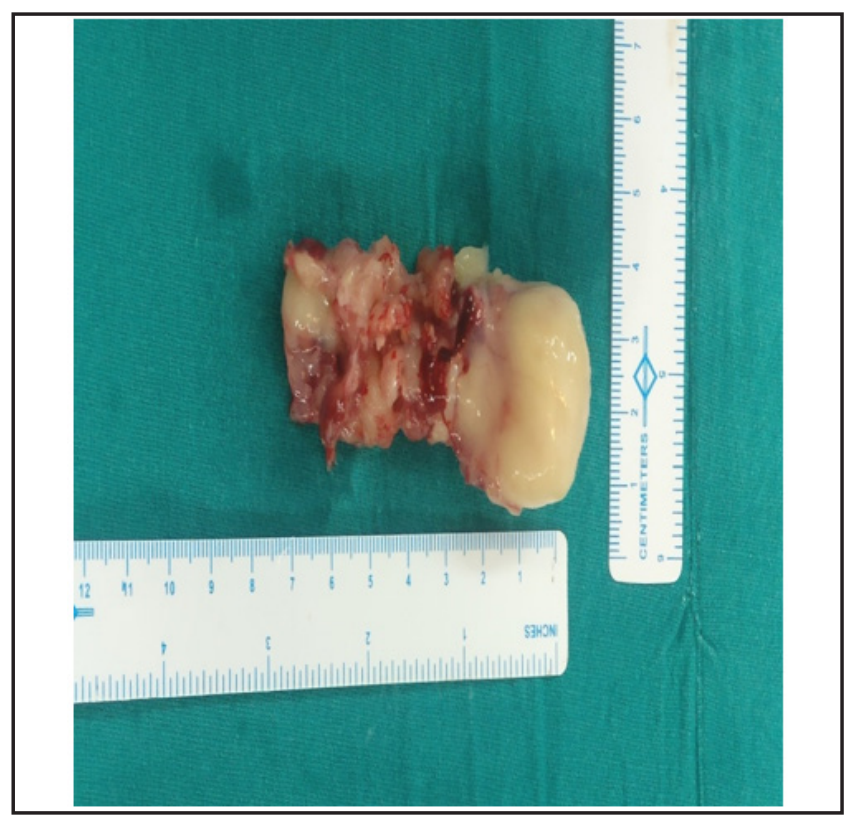

Fig. 4a. Gross specimen

A differential diagnosis was made of benign nasal mass, sphenochoanal polyp and inverted papilloma, on the basis of unilateral disease and the clinical features. The patient was planned for endoscopic excision under general anaesthesia (GA). Informed written consent was taken. Under GA, nasal decongestion was done. Diagnostic nasal endoscopy was done which revealed left concha bullosa. A polypoidal mass of approximate size $7 \times 3 \mathrm{~cm}$ was seen arising from left sphenoethmoidal recess and occupying the whole of choana (Fig. 3). An additional Ostia was seen in the anterior wall of sphenoid sinus. Concha bullosa reduction was done. The polypoidal mass was traced to its origin till the sphenoethmoidal recess and removed with the help of coblator. Wide sphenoidotomy was done and polypoidal tissue was removed from the posterior ethmoids (Fig. 4a). Haemostastis was achieved and no postoperative nasal packing was required. The postoperative period was uneventful and the patient was discharged on postoperative day 2 and was followed up with the biopsy report. Final histopathological examination revealed biphasic tumour with hypercellular (Antoni A) and hypocellular (Antoni B) areas. (Fig. 4b). An impression of schwannoma was made.

In the one month follow up of the patient, the

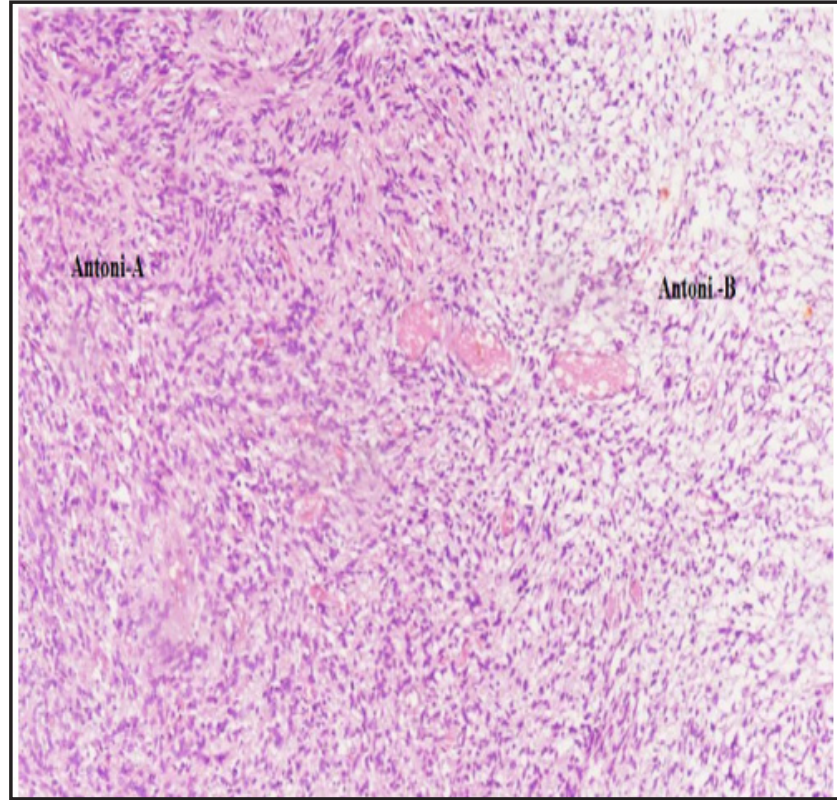

Fig. 4b. Microscopic picture (H\&E, 100X)

nasal cavity had re-epithelised and was healthy. The patient was asymptomatic and computed tomography scan showed complete clearance of disease with no recurrence (Fig. 5).

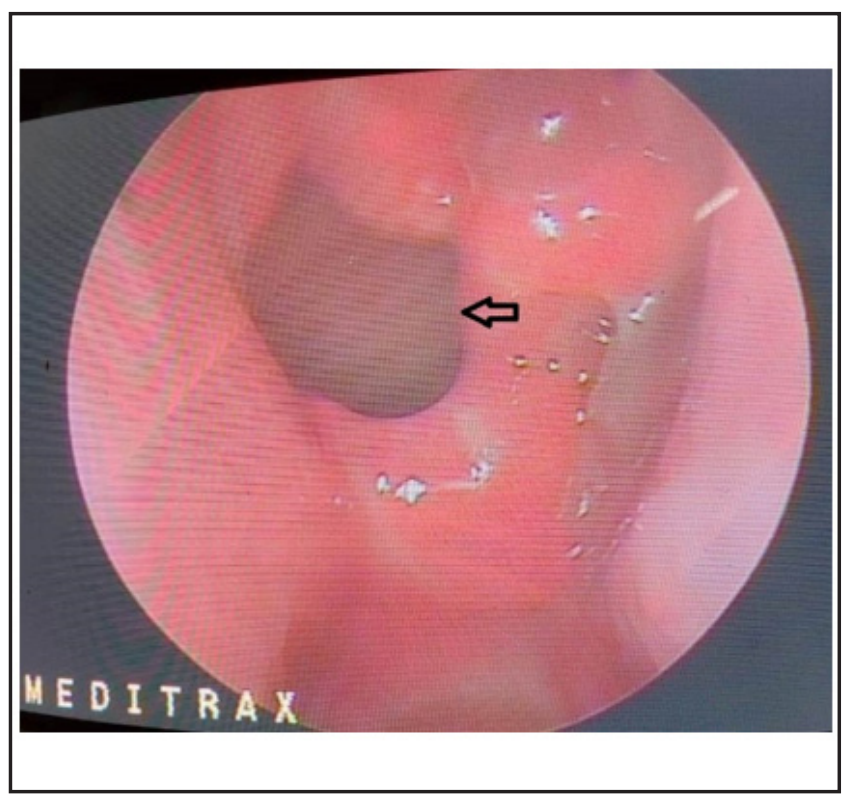

Fig. 5- Post operative endoscopy of the patient showing healthy cavity (Black ar-row) 


\section{Discussion}

Schwannomas, also known as neurilemomas, are benign, slow growing, encapsulated tumours of the peripheral nervous system, arising from Schwann cells. They were first described by Verocay in 1908. The literature mentions very few cases of nose and paranasal sinus schwannomas that are mostly seen in adults aged 40-60 years and without gender or racial predilection. ${ }^{2}$ It commonly involves the ethmoid sinus, maxillary sinus, the nasal cavity, sphenoid sinus and nasal septum in that order of decreasing prevalence. ${ }^{3}$ They usually present with nasal obstruction, pain, headache, nasal bleed. They may also present with ptosis, proptosis or diplopia, pain or paraesthesias. Rare cases of intracranial and intraorbital extension have been studied, which showed symptoms of rhi-norrhea, decreased level of consciousness, and strabismus. ${ }^{4}$ Association with neurofibromatosis type-2 (NF-2) is seen in cases where multiple schwannomas occur. Imaging features are generally nonspecific but mild enhancement may be evident on contrast CT. Bone remodeling may also be appreciated. MR imaging shows intermediate T1 and variable T2 signal intensity. ${ }^{5}$ Histopathology is considered as the gold standard for diagnosis. Macroscopically, schwannomas appear as cystic or gelatinous, well encapsulated masses. Microscopically, they can be classified into:

1. ANTONI A - which comprises of organized compact stroma with spindle cells and parallel rows of palisading nuclei

2. ANTONI B - which has a disorganized loose myxoid stroma with few spindle cells

Nasal schwannomas can be differentiated from those arising elsewhere, by their histopathology as they are hyper-cellular and do not have fibrous encapsulation.

Schwannomas can be graded by the Enneking system based on their severity. This system classi-fied the tumour into three grades. Grade 1 includes lesions that are inactive, Grade 2 are the le-sions that deform surrounding tissue but are not locally destructive while grade 3 encompasses those lesions that are locally aggressive but do not have metastatic potential. ${ }^{6}$

The differential diagnosis for the nasal mass may include: concha bullosa, antrochoanal polyp, in- verted papilloma, lymphoma, capillary hemangioma, mucocoele, fibrous dysplasia,meningioma, neurofibroma, enchondroma,dermoid cyst, idiopathic midline granuloma, melanoma, myxoma, fibromyxoma, squamous cell carcinoma, esthesioneuroblastoma, chondrosarcoma, and intranasal extension of the nasopharyngeal angiofibroma. ${ }^{7,8}$

In this patient, a provisional diagnosis of benign nasal mass was made due to the unilateral presen-tation of disease as well as presence of symptoms like nasal obstruction and unilateral nasal bleed. Intra-operatively, a polypoidal mass was seen in the left nasal cavity, pointing more towards a spheno-choanal polyp. The mass was revealed to be a schwannoma, kept low on the list of differ-entials due to its rare occurrence, on histopathological examination. Definite treatment for schwannomas is surgery, which can be either endoscopic or open. This is decided by the operating surgeon after careful consideration of the site and extent of the disease. Recurrence of the disease post operatively is rare. ${ }^{9}$ However malignant changes may occur in long standing lesions. ${ }^{10}$

Although a rare entity, schwannomas should be considered as a differential in cases of unilateral nasal obstruction and nasal bleed in patients. Confirmatory diagnosis can only be made on the ba-sis of histopathology with complete surgical excision being the treatment of choice.

\section{References}

1. Gupta M, Rao N, Kour C, Kaur I. Septal Schwannoma of the Nose: A Rare Case. Turk Arch Otorhinolaryngol. 2017; 55(1):41-3

2. Pauna HF, de Carvalho GM, Guimarães AC, Maunsell RCK, Sakano E. Schwannoma of the nasal septum: evaluation of unilateral nasal mass. Braz J Otorhinolaryngol. 2013; 79(3):403

3. Calcaterra TC, Rich JR, Ward PW. Neurilemoma of the Sphenoid Sinus. Arch Otolaryngol - Head Neck Surg. 1974;100(5):383-5

4. Fujiyoshi F, Kajiya Y, Nakajo M. CT and MR imaging of nasoethmoid schwannoma with in-tracranial extension. Am J Roentgenol. 1997;169(6):1754-5

5. Yu E, Mikulis D, Nag S. CT and MR imaging findings in sinonasal schwannoma. Am J Neuroradiol. 2006; 27(4):929-30

6. Campanacci M. Bone and Soft Tissue Tumors: Clinical Features, Imaging, Pathology and Treatment. 2nd ed. Vienna: 
Springer; 1999

7. Kim YS, Kim H-J, Kim C-H, Kim J. CT and MR Imaging Findings of Sinonasal Schwannoma: A Review of 12 Cases. Am J Neuroradiol. 2013; 34(3):628-33

8. Valencia MP, Castillo M. Congenital and Acquired Lesions of the Nasal Septum: A Practical Guide for Differential Diagnosis. RadioGraphics 2008 Jan;28(1):205-23
9. Mey KH, Buchwald C, Daugaard S, Prause JU. Sinonasal schwannoma--a clinicopathological analysis of five rare cases. Rhinology 2006; 44(1):46-52

10. Kurtkaya-Yapicier Ö, Scheithauer B, Woodruff JM. The pathobiologic spectrum of Schwannomas. Histol Histopathol. 2003; (18):925-34. 